\title{
The Effects of Gravity of an Loss-of-Equilibrium CME Initiation Model
}

\author{
Katharine K. Reeves and Terry G. Forbes \\ University of New Hampshire, Institute for Earth, Oceans and Space, Durham, NH 03824, \\ USA \\ email: kreeves@unh.edu
}

\begin{abstract}
We include gravity in a loss of equilibrium model for the initiation of coronal mass ejections (CMEs). We examine equilibria for both normal and inverse polarity and neglect the effects of current sheets. Although equilibria exist for normal polarities, in the absence of current sheets, the equilibria are unstable to horizontal perturbations. For the inverse polarity configuration, we find that gravity generally has a negligible effect if the magnetic field is strong $(>50 \mathrm{G})$ but that it can have a significant effect if the magnetic field is weak. Specifically, if the characteristic magnetic field is less than about $6 \mathrm{G}$, no eruption occurs if the CME mass is on the order of $2 \times 10^{16} \mathrm{gm}$.
\end{abstract}

Keywords. Sun: coronal mass ejections, MHD

\section{Introduction}

Loss of equilibrium CME models are characterized by a catastrophic unbalancing of the forces on a flux rope in the solar corona. In this paper, we will examine the effect of including gravity in a system that consists of a flux rope at a height $h$ above the corona and two line sources embedded in the photosphere a distance $2 \lambda$ apart. There are photospheric currents that are accounted for by an image current submerged a distance $h$ below the photosphere. This magnetic configuration has been examined before (Forbes \& Priest (1995), Lin \& Forbes (2000)), but without the inclusion of gravity in the equilibrium equation.

The effect of gravity was previously considered by Isenberg, Forbes \& Démoulin (2002) for a 2D quadrupolar configuration, but only for the inverse configuration. They determined that in the asymptotic case of an infinitely small flux rope radius, there is a limiting magnetic field below which no loss of equilibrium occurs. A similar result is suggested in Lin (2004), for an inverse configuration containing a current sheet. In this paper, we will examine the effect of gravity on the equilibria of both normal and inverse configurations (see Figure 1), and we will examine the equilibrium curves with respect to footpoint motions to determine the conditions under which a loss of equilibrium is possible for configurations with no current sheet and a finite flux rope radius.

We also examine the equilibria with respect to draining mass from the flux rope. Mass loss has been suggested as a mechanism for CME initiation (e.g. Zhang \& Low (2004)), and it becomes a factor in the balance of forces on the flux rope only when gravity is taken in to account. The other variations of this model that include the effects of gravity (Isenberg, Forbes \& Démoulin (2002), Lin (2004)) do not examine the equilibrium curves that result when the mass of the flux rope is varied. 

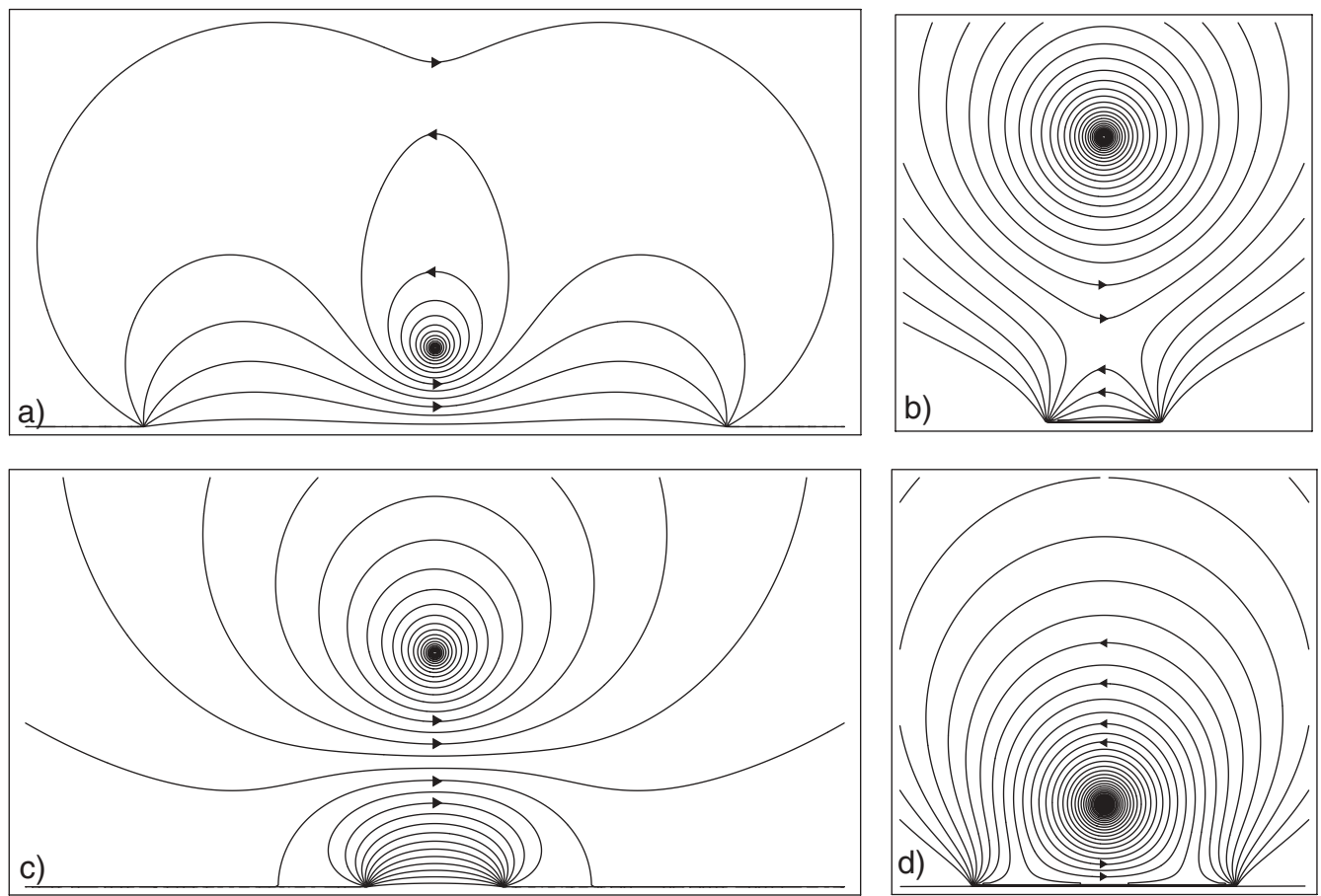

Figure 1. Magnetic configurations used in the model. Panels a) and b) are the normal and inverse polarity configurations, respectively, including an x-line. Panels c) and d) are the normal and inverse configurations, respectively, with no x-line. Arrows indicate magnetic field direction.

\section{Equilibrium curves}

\subsection{Governing equations}

The inverse and normal polarity magnetic configurations used in these calculations are shown in Figure 1. Some configurations do not have $\mathrm{x}$-lines - the equation for the $\mathrm{x}$-line location is given in Priest \& Forbes (2000), and it can be shown that the x-line disappears in the normal configuration when $h / \lambda>-1 / 2 J$, where $J$ is the normalized current in the flux rope. For the inverse configuration, no x-point occurs if $h / \lambda<2 J$.

The equilibrium curves are curves in a parameter space where the total force on the flux rope is zero. In the absence of a current sheet, the vertical forces on the flux rope are: an upward force due to the image current, a downward force due to gravity, and a force due to the photospheric line sources which is either upwards (normal) or downwards (inverse). Normal polarity configurations can be in equilibrium only if gravity is included, since all other vertical forces on the flux rope in that configuration are upwards. In general, the equation for the vertical force per unit length on the flux rope is

$$
F_{y}=\frac{I B_{\text {ext }_{x}}(x, y)}{c}-\frac{m g_{0}}{\left(1+h / R_{\text {sun }}\right)}
$$

where $I$ is the current in the flux rope, $B_{\text {ext }}$ is the external field, $m$ is the mass of the flux rope per unit length, $g_{0}$ is the value of gravity at the sun's surface and $R_{\text {sun }}$ is the radius of the sun. The $x$ component of the external field is given by

$$
B_{\text {ext }}(x, y)=\frac{2 A_{0}}{\pi}\left[\frac{J(y+h)}{x^{2}+(y+h)^{2}}-\frac{2 \lambda\left(\lambda^{2}-x^{2}+y^{2}\right)}{\lambda^{4}-2 \lambda^{2}\left(x^{2}-y^{2}\right)+\left(x^{2}+y^{2}\right)^{2}}\right]
$$



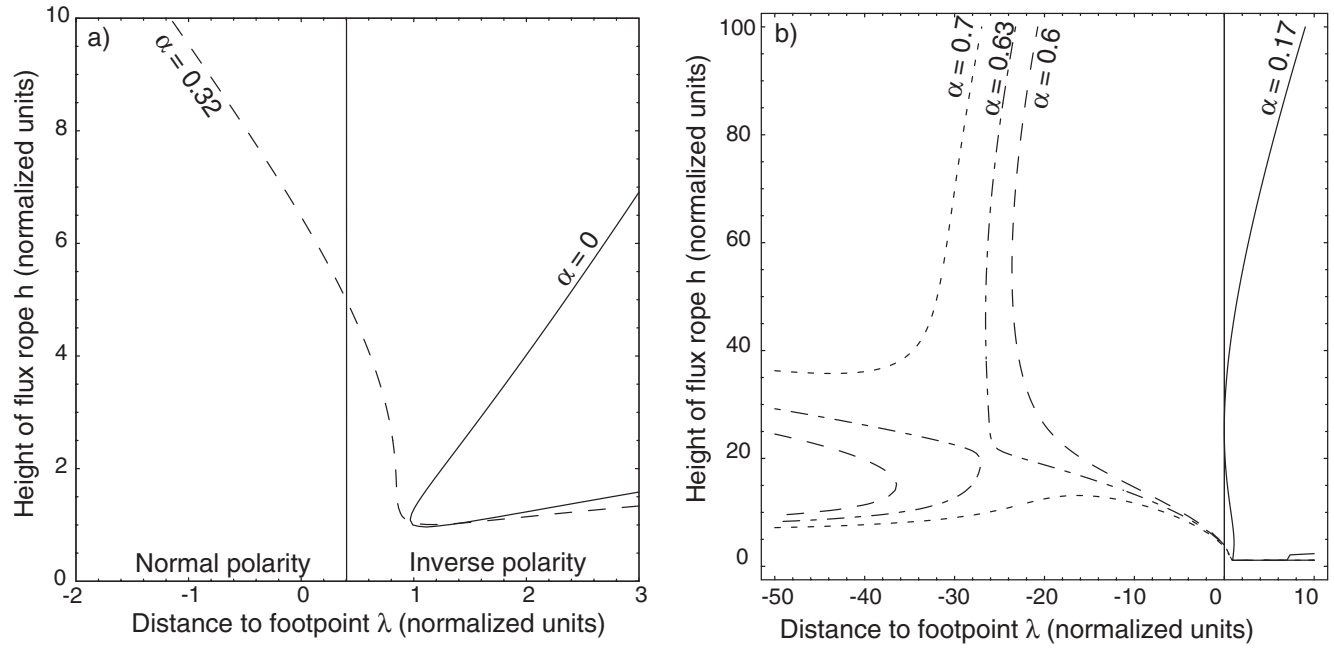

Figure 2. Equilibrium curves with respect to footpoint separation that have nose points a) in the inverse configuration and b) in the normal configuration. Negative values of $\lambda$ indicate normal polarity magnetic configurations, while positive values indicate inverse polarity configurations. Distances are normalized to the height of the flux rope at the maximum current point.

where $A_{0}$ is the value of the vector potential at the footpoints, and $J=I \pi / c A_{0}$. The first term in $B_{\text {ext }}$ is due to the image current and the second is due to the photospheric source terms.

The horizontal force on the flux rope depends only on the photospheric line sources, since the image current always lies directly under the flux rope:

$$
F_{x}=\frac{I B_{e t_{y}}(x, y)}{c}=-\frac{A_{0}}{\pi}\left[\frac{4 x y \lambda}{\lambda^{4}-2 \lambda^{2}\left(x^{2}-y^{2}\right)+\left(x^{2}+y^{2}\right)^{2}}\right]
$$

\subsection{Footpoint motions}

Figure 2 shows select equilibrium curves with respect to footpoint motions for different values of $\alpha=m g_{0} h_{0} c^{2} / I_{0}^{2}$, a parameter that relates the strength of the gravitational force to the force of the characteristic magnetic field. A loss of equilibrium occurs at the point where the equilibrium curve folds back on itself, creating a "nose point" - an example can be clearly seen for the zero gravity case in Figure 2a. If the force due to the characteristic field is weak enough compared to the force of gravity, this nose point can disappear, as shown for the $\alpha=0.32$ case in Figure 2a. In real units, if the mass per unit length, $m$ is $2.1 \times 10^{6} \mathrm{gm} / \mathrm{cm}$ and $h_{0}=5 \times 10^{9} \mathrm{~cm}$, then the characteristic field (given by $\left.I_{0} / c h_{0}\right)$ must be greater than $6 \mathrm{G}$ for a nose point to exist in the inverse configuration in the resulting equilibrium curve.

For normal field configurations, there are no equilibria possible if $\alpha<0.17$, as shown in Figure 2b. For larger values of $\alpha$, equilibria do exist in the normal field configuration, and there are nose points. All nose points vanish, however, when $\alpha>0.65$. For the same values of $m$ and $h_{0}$ used above, the characteristic field should be between 4.2 and 8.2 $\mathrm{G}$ for nose points to exist in the normal configuration. It should be noted, as Jun Lin pointed out in the discussion of this paper, that the normal equilibria calculated here are not stable with respect to horizontal perturbations even though some branches are stable with respect to vertical perturbations. Stability could possibly achieved with the introduction of a current sheet at the $\mathrm{x}$-line for those configurations that have $\mathrm{x}$-lines (see Figure 1). 

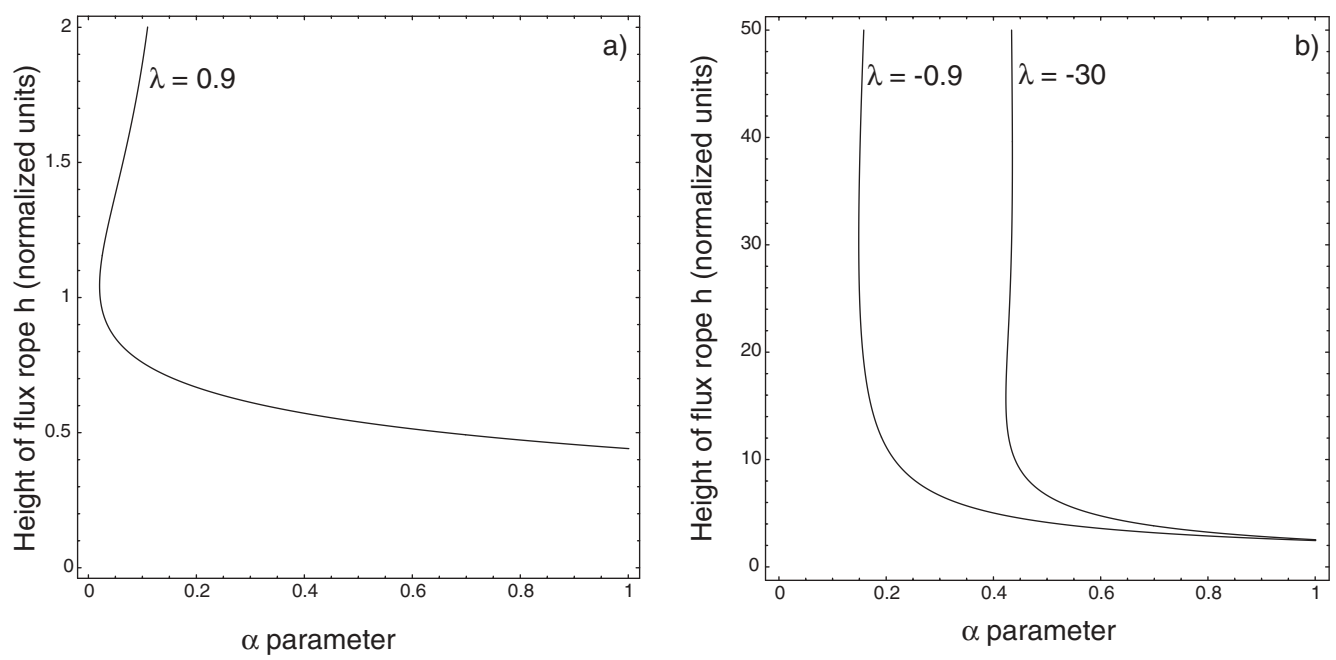

Figure 3. Equilibrium curves with respect to mass loss in the prominence showing the location of nose points for a) the inverse configuration and b) the normal configuration. Distances are normalized to an arbitrary point on the equilibrium curve, since there is no maximum current point.

\subsection{Mass loss}

Figure 3 shows equilibrium curves for inverse and normal polarity configurations with respect to a decrease in the parameter $\alpha$, which is equivalent to mass loss in the flux rope since $\alpha$ is directly proportional to the mass. The nose point in the inverse polarity case occurs at a smaller value of $\alpha$ than either of the two normal polarity cases. Thus there must be more mass drained from an inverse configuration flux rope to bring it to a catastrophic loss of equilibrium than for a flux rope in the normal configuration. This finding agrees with Zhang \& Low (2004), who estimated masses necessary for anchoring the flux rope in the corona and found that more mass is needed to keep the normal configuration from erupting.

Figure 4 shows the total energy plotted along the mass loss equilibrium curves for three values of $\lambda$. The $\mathrm{x}$ on each curve marks the position where the loss of equilibrium occurs. Zhang \& Low (2004) have contended that normal magnetic configurations store more energy than inverse magnetic configurations, but these results show that it is not always the case. Specifically, normal configurations with large footpoint separations have a higher total energy at the loss of equilibrium point than inverse configurations, but normal topologies with close together footpoints have a lower energy at the loss of equilibrium point. The configuration with large footpoint separation is pushing the boundaries of our model, however, because we use a gravity term that falls off as $1 / h^{2}$ in combination with an infinite plane cartesian geometry.

\section{Conclusions}

We have found that when equilibria with respect to footpoint separation are examined, the nose points in the equilibrium curves can disappear for both normal and inverse polarities if the force on the flux rope due to the characteristic magnetic field is weak compared to the force of gravity. The minimum magnetic field that allows eruptions to occur in the inverse case is approximately $6 \mathrm{G}$. This value is lower than the $17 \mathrm{G}$ found in Isenberg, Forbes \& Démoulin (2002), possibly because of the different magnetic 


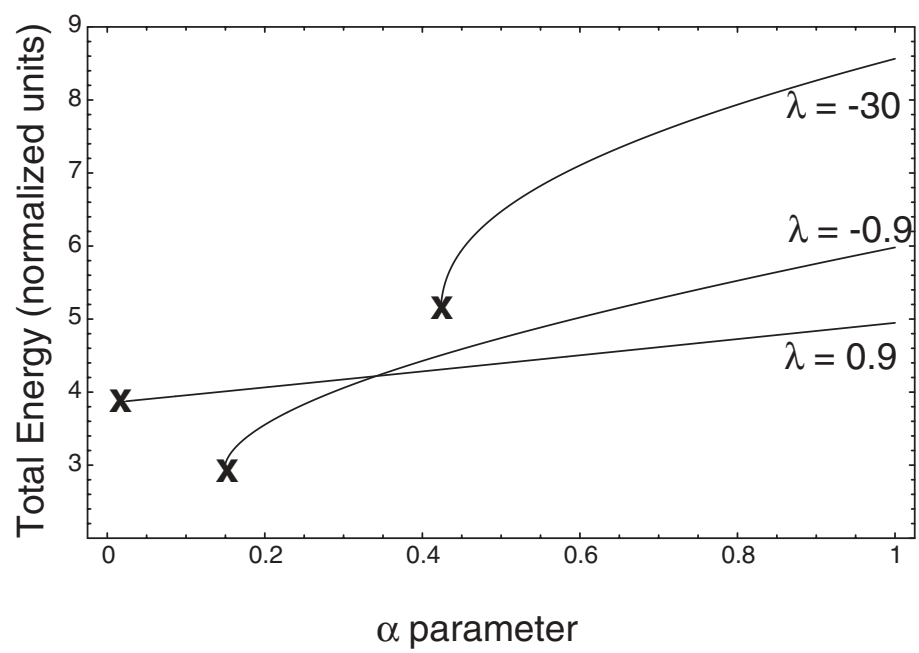

Figure 4. Plots of the total energy along the mass loss equilibrium curves for three different values of $\lambda$. Positive values of $\lambda$ indicate inverse configurations and negative values indicate normal configurations. The position of the loss of equilibrium is marked with an $\mathrm{x}$.

configurations used. Lin (2004) finds a minimum value for the characteristic field of 13.5 $\mathrm{G}$, which is higher than our value possibly because he is concerned with CME propagation rather than the actual disappearance of the nose point. When a current sheet is included, as in Lin (2004), there could be parameters for which the CME does not propagate out in to the corona, but a loss of equilibrium still occurs.

We have also examined the equilibria with respect to mass loss in the flux rope, and we find that more mass must drain to attain a loss of equilibrium in the inverse configuration than in the normal configuration, which agrees with the conclusions of Zhang \& Low (2004). Plotting the energy along the mass loss equilibrium curves for several different values of $\lambda$, we find that the total energy at the nose point can be higher in the normal configuration than in the inverse configuration if $\lambda$ is large enough, however, this conclusion is weakened by the constraints of our model. For small values of $\lambda$ the energy at the loss of equilibrium point is smaller in the normal configuration than in the inverse configuration, contradicting the assertion of Zhang \& Low (2004) that normal configurations store more energy than inverse ones.

\section{Acknowledgements}

This work was supported by NSF grant ATM-0327512 and NASA LWS TR+T grant NNH05AA13I to UNH as well as DoD MURI grants to UC Berkeley and the University of Michigan.

\section{References}

Forbes, T.G. \& Priest, E.R. 1995, ApJ 446, 377

Isenberg, P.A., Forbes, T.G. \& Démoulin, P. 1993, ApJ 417, 368

Lin, J. \& Forbes T.G. 2000, J. Geophys. Res. 105, 2375

Lin, J. 2004, Sol. Phys., 602, 422

Priest, E.R. \& Forbes, T.G. 2000, Magnetic Reconnection (Cambridge University Press), p.378

Zhang, M. \& Low, B. C. 2004, ApJ 600, 1043 


\section{Discussion}

Koutchmy: Could your flux rope correspond to the cavity we see rising up during a CME possibly due to buoyancy?

REEVES: No, in this model the radius of the flux rope is very small for reasons of mathematical simplicity.

JUN LIN: We might need to worry about the stability of the system to the horizontal disturbance in the normal polarity case.

REEves: This point is an extremely good one and we will examine the stability of the normal polarity equilibria with respect to horizontal perturbations.

GOPALSWAmy: Is your stability analysis consistent with the observational fact that inverse polarity filaments are more common than the normal polarity ones?

ReEves: Yes, especially in light of Jun Lin's comment about stability with respect to perturbations in the horizontal direction. Also, there are no equilibria in the normal configuration when the fields are strong compared to the gravitational force on the flux rope, but there are inverse equilibria in this case. 